\title{
THE RADIUS OF $\alpha$-CONVEXITY OF NORMALIZED BESSEL FUNCTIONS OF THE FIRST KIND
}

\author{
ÁRPÁD BARICZ, HALIT ORHAN, AND RÓBERT SZÁSZ
}

\begin{abstract}
The radii of $\alpha$-convexity are deduced for three different kind of normalized Bessel functions of the first kind and it is shown that these radii are between the radii of starlikeness and convexity, when $\alpha \in[0,1]$, and they are decreasing with respect to the parameter $\alpha$. The results presented in this paper unify some recent results on the radii of starlikeness and convexity for normalized Bessel functions of the first kind. The key tools in the proofs are some interlacing properties of the zeros of some Dini functions and the zeros of Bessel functions of the first kind.
\end{abstract}

\section{Introduction and the main results}

Let $\mathbb{D}(0, r)$ be the open disk $\{z \in \mathbb{C}:|z|<r\}$, where $r>0$, and set $\mathbb{D}=\mathbb{D}(0,1)$. By $\mathcal{A}$ we mean the class of analytic functions $f: \mathbb{D}(0, r) \rightarrow \mathbb{C}$ which satisfy the usual normalization conditions $f(0)=$ $f^{\prime}(0)-1=0$. Denote by $\mathcal{S}$ the class of functions belonging to $\mathcal{A}$ which are univalent in $\mathbb{D}(0, r)$ and let $\mathcal{S}^{*}(\beta)$ be the subclass of $\mathcal{S}$ consisting of functions which are starlike of order $\beta$ in $\mathbb{D}(0, r)$, where $0 \leq \beta<1$. The analytic characterization of this class of functions is

$$
\mathcal{S}^{*}(\beta)=\left\{f \in \mathcal{S}: \operatorname{Re}\left(\frac{z f^{\prime}(z)}{f(z)}\right)>\beta \text { for all } z \in \mathbb{D}(0, r)\right\},
$$

while the real number

$$
r_{\beta}^{*}(f)=\sup \left\{r>0: \operatorname{Re}\left(\frac{z f^{\prime}(z)}{f(z)}\right)>\beta \quad \text { for all } z \in \mathbb{D}(0, r)\right\}
$$

is called the radius of starlikeness of order $\beta$ of the function $f$. Note that $r^{*}(f)=r_{0}^{*}(f)$ is the largest radius such that the image region $f\left(\mathbb{D}\left(0, r^{*}(f)\right)\right)$ is a starlike domain with respect to the origin. Also, let $\mathcal{K}(\beta)$ be the subclass of $\mathcal{S}$ consisting of functions which are convex of order $\beta$ in $\mathbb{D}(0, r)$, where $0 \leq \beta<1$. The well-known analytic characterization of this class of functions is

$$
\mathcal{K}(\beta)=\left\{f \in \mathcal{S}: \operatorname{Re}\left(1+\frac{z f^{\prime \prime}(z)}{f^{\prime}(z)}\right)>\beta \text { for all } z \in \mathbb{D}(0, r)\right\}
$$

and the real number

$$
r_{\beta}^{c}(f)=\sup \left\{r>0: \operatorname{Re}\left(1+\frac{z f^{\prime \prime}(z)}{f^{\prime}(z)}\right)>\beta \quad \text { for all } z \in \mathbb{D}(0, r)\right\}
$$

is called the radius of convexity of order $\beta$ of the function $f$. Note that $r^{c}(f)=r_{0}^{c}(f)$ is the largest radius such that the image region $f\left(\mathbb{D}\left(0, r^{c}(f)\right)\right)$ is a convex domain with respect to the origin. Furthermore, let $\mathcal{M}(\alpha, \beta)$ be the subclass of $\mathcal{S}$ consisting of functions which are $\alpha$-convex of order $\beta$ in $\mathbb{D}(0, r)$, where $\alpha \in \mathbb{R}$ and $0 \leq \beta<1$. The analytic characterization of this class of functions is

$$
\mathcal{M}(\alpha, \beta)=\left\{f \in \mathcal{S}: \operatorname{Re}\left((1-\alpha) \frac{z f^{\prime}(z)}{f(z)}+\alpha\left(1+\frac{z f^{\prime \prime}(z)}{f^{\prime}(z)}\right)\right)>\beta \text { for all } z \in \mathbb{D}(0, r)\right\},
$$

while the real number

$$
r_{\alpha, \beta}(f)=\sup \left\{r>0: \operatorname{Re}\left((1-\alpha) \frac{z f^{\prime}(z)}{f(z)}+\alpha\left(1+\frac{z f^{\prime \prime}(z)}{f^{\prime}(z)}\right)\right)>\beta \quad \text { for all } z \in \mathbb{D}(0, r)\right\}
$$

is called the radius of $\alpha$-convexity of order $\beta$ of the function $f$. The radius of $\alpha$-convexity of order $\beta$ is the generalization of the radius of starlikeness of order $\beta$ and of the radius of convexity of order $\beta$. We

File: alpha.tex, printed: 2018-7-04, 8.22

2010 Mathematics Subject Classification. 33C10, 30C45.

Key words and phrases. Normalized Bessel functions of the first kind; convex functions; starlike functions; $\alpha$-convex functions; radius of convexity; radius of starlikeness; radius of $\alpha$-convexity; Dini function; minimum principle for harmonic functions; zeros of Bessel functions. 
have $r_{0, \beta}(f)=r_{\beta}^{*}(f)$ and $r_{1, \beta}(f)=r_{\beta}^{c}(f)$. For more details on starlike, convex and $\alpha$-convex functions we refer to [10, 12, 13] and to the references therein.

The Bessel function of the first kind of order $\nu$ is defined by [14, p. 217]

$$
J_{\nu}(z)=\sum_{n \geq 0} \frac{(-1)^{n}}{n ! \Gamma(n+\nu+1)}\left(\frac{z}{2}\right)^{2 n+\nu}, \quad z \in \mathbb{C} .
$$

In this paper we focus on the following normalized forms

$$
\begin{gathered}
f_{\nu}(z)=\left(2^{\nu} \Gamma(\nu+1) J_{\nu}(z)\right)^{\frac{1}{\nu}}=z-\frac{1}{4 \nu(\nu+1)} z^{3}+\ldots, \nu \neq 0, \\
g_{\nu}(z)=2^{\nu} \Gamma(\nu+1) z^{1-\nu} J_{\nu}(z)=z-\frac{1}{4(\nu+1)} z^{3}+\frac{1}{32(\nu+1)(\nu+2)} z^{5}-\ldots, \\
h_{\nu}(z)=2^{\nu} \Gamma(\nu+1) z^{1-\frac{\nu}{2}} J_{\nu}(\sqrt{z})=z-\frac{1}{4(\nu+1)} z^{2}+\ldots,
\end{gathered}
$$

where $\nu>-1$. We note that in fact for $z \in \mathbb{C} \backslash\{0\}$ we have

$$
f_{\nu}(z)=\exp \left(\frac{1}{\nu} \log \left(2^{\nu} \Gamma(\nu+1) J_{\nu}(z)\right)\right),
$$

where Log represents the principal branch of the logarithm, and in this paper every multi-valued function is taken with the principal branch. We also mention that the univalence, starlikeness and convexity of Bessel function of the first kind were studied extensively in several papers. We refer to [1, 2, 3, 4, 5, 6, 7, 8, 9, 11, 15, 16] and to the references therein.

In this paper we make a further contribution to the subject by showing the following new sharp results contained in Theorems 1, 2 and 3. The proofs of Theorems 1, 2 and 3 can be found in section 2.

Theorem 1. If $\nu>0, \alpha \geq 0$ and $\beta \in[0,1)$, then the radius of $\alpha$-convexity of order $\beta$ of the function $f_{\nu}$ is the smallest positive root of the equation

$$
\alpha\left(1+\frac{r J_{\nu}^{\prime \prime}(r)}{J_{\nu}^{\prime}(r)}\right)+\left(\frac{1}{\nu}-\alpha\right) \frac{r J_{\nu}^{\prime}(r)}{J_{\nu}(r)}=\beta .
$$

The radius of $\alpha$-convexity satisfies $r_{\alpha, \beta}\left(f_{\nu}\right) \leq j_{\nu, 1}^{\prime}<j_{\nu, 1}$, where $j_{\nu, 1}$ and $j_{\nu, 1}^{\prime}$ denote the first positive zeros of $J_{\nu}$ and $J_{\nu}^{\prime}$, respectively. Moreover, the function $\alpha \mapsto r_{\alpha, \beta}\left(f_{\nu}\right)$ is strictly decreasing on $[0, \infty)$ and consequently we have $r_{\beta}^{c}\left(f_{\nu}\right)<r_{\alpha, \beta}\left(f_{\nu}\right)<r_{\beta}^{*}\left(f_{\nu}\right)$ for all $\alpha \in(0,1), \beta \in[0,1)$ and $\nu>0$.

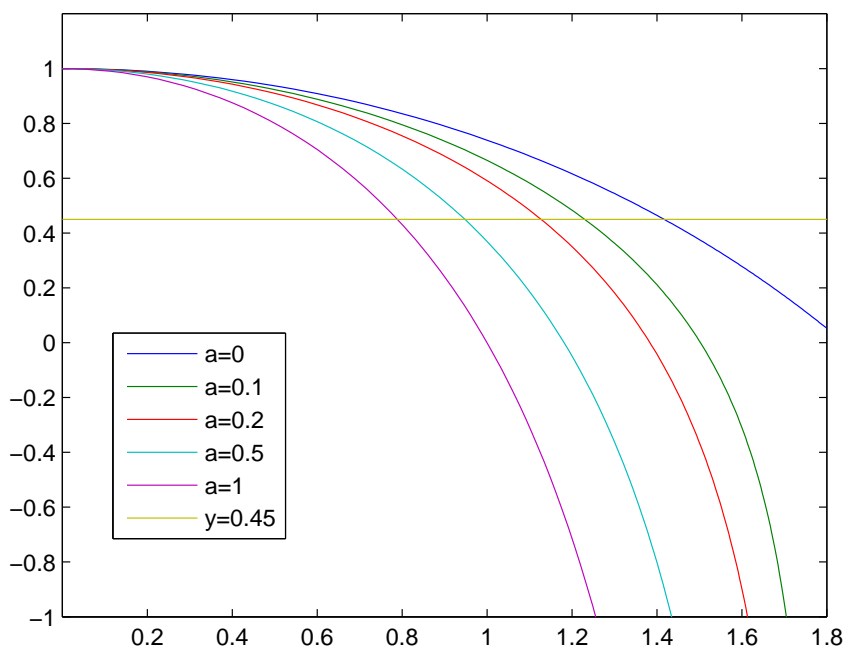

Figure 1. The graph of the function $r \mapsto \mathbb{J}\left(a, f_{1}(r)\right)$ for $a \in\{0,0.1,0.2,0.5,1\}$ on $[0,1.8]$.

It is worth to mention that the cases $\alpha=0$ and $\alpha=1$ of the above Theorem were considered recently in 4, Theorem 1(a)] and [6, Theorem 1.1]. Our Theorem 1] is a common generalization of these results. 
Figure 1 illustrates the fact that if $\alpha \in[0,1]$, then the radius of $\alpha$-convexity of the function $f_{\nu}$ is between its radii of convexity and starlikeness, that is, $r_{\beta}^{c}\left(f_{\nu}\right)<r_{\alpha, \beta}\left(f_{\nu}\right)<r_{\beta}^{*}\left(f_{\nu}\right)$ for all $\alpha \in(0,1), \beta \in[0,1)$ and $\nu>0$. We considered the particular cases when $\beta=0.45, \nu=1$ and $\alpha \in\{0,0.1,0.2,0.5,1\}$.

Theorem 2. If $\nu>-1, \alpha \geq 0$ and $\beta \in[0,1)$, then the radius of $\alpha$-convexity of order $\beta$ of the function $g_{\nu}$ is the smallest positive root of the equation

$$
1+(\alpha-1) \frac{r J_{\nu+1}(r)}{J_{\nu}(r)}+\alpha r \frac{r J_{\nu+2}(r)-3 J_{\nu+1}(r)}{J_{\nu}(r)-r J_{\nu+1}(r)}=\beta
$$

The radius of $\alpha$-convexity satisfies $r_{\alpha, \beta}\left(g_{\nu}\right) \leq \alpha_{\nu, 1}<j_{\nu, 1}$, where $\alpha_{\nu, 1}$ is the first positive zero of the Dini function $z \mapsto(1-\nu) J_{\nu}(z)+z J_{\nu}^{\prime}(z)$. Moreover, the function $\alpha \mapsto r_{\alpha, \beta}\left(g_{\nu}\right)$ is strictly decreasing on $[0, \infty)$ and consequently we have $r_{\beta}^{c}\left(g_{\nu}\right)<r_{\alpha, \beta}\left(g_{\nu}\right)<r_{\beta}^{*}\left(g_{\nu}\right)$ for all $\alpha \in(0,1), \beta \in[0,1)$ and $\nu>-1$.

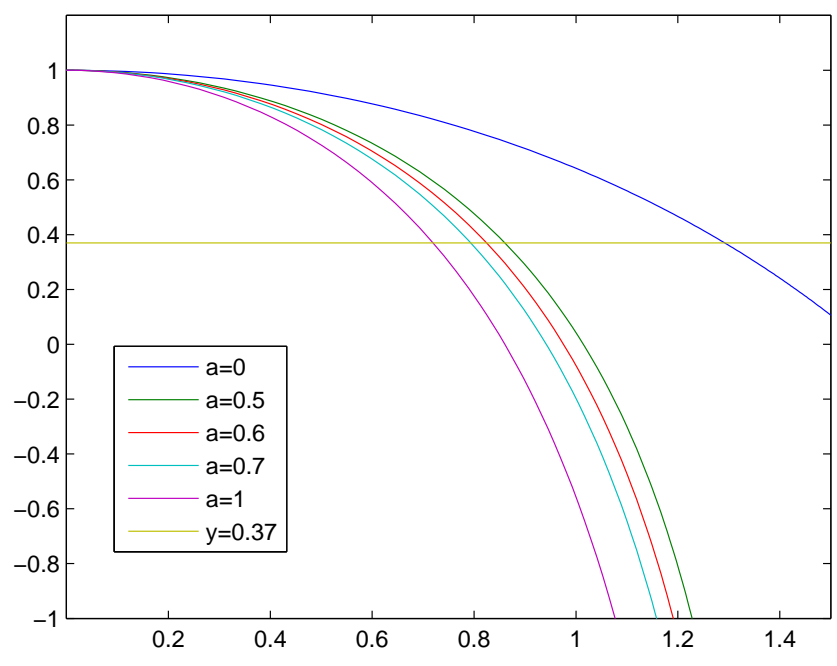

FigurE 2. The graph of the function $\left.r \mapsto \mathbb{J}\left(a, g_{0.5} r\right)\right)$ for $a \in\{0,0.5,0.6,0.7,1\}$ on $[0,1.5]$.

We also note that the cases $\alpha=0$ and $\alpha=1$ of the above Theorem were considered recently in [4, Theorem 1(b)] and [6, Theorem 1.2]. Our Theorem 2 is a common generalization of these results. Figure 2 illustrates the fact that when $\alpha \in[0,1]$ the radius of $\alpha$-convexity of the function $g_{\nu}$ is between its radii of convexity and starlikeness, that is, $r_{\beta}^{c}\left(g_{\nu}\right)<r_{\alpha, \beta}\left(g_{\nu}\right)<r_{\beta}^{*}\left(g_{\nu}\right)$ for all $\alpha \in(0,1), \beta \in[0,1)$ and $\nu>-1$. We considered the particular cases when $\beta=0.37, \nu=0.5$ and $\alpha \in\{0,0.5,0.6,0.7,1\}$.

Theorem 3. If $\nu>-1, \alpha \geq 0$ and $\beta \in[0,1)$, then the radius of $\alpha$-convexity of order $\beta$ of the function $h_{\nu}$ is the smallest positive root of the equation

$$
(1-\alpha)\left(1-\frac{r^{\frac{1}{2}}}{2} \cdot \frac{J_{\nu+1}\left(r^{\frac{1}{2}}\right)}{J_{\nu}\left(r^{\frac{1}{2}}\right)}\right)+\alpha\left(1+\frac{r^{\frac{1}{2}}}{2} \cdot \frac{r^{\frac{1}{2}} J_{\nu+2}\left(r^{\frac{1}{2}}\right)-4 J_{\nu+1}\left(r^{\frac{1}{2}}\right)}{2 J_{\nu}\left(r^{\frac{1}{2}}\right)-r^{\frac{1}{2}} J_{\nu+1}\left(r^{\frac{1}{2}}\right)}\right)=\beta .
$$

The radius of $\alpha$-convexity satisfies $r_{\alpha, \beta}\left(h_{\nu}\right) \leq \beta_{\nu, 1}^{2}<j_{\nu, 1}^{2}$, where $\alpha_{\beta, 1}$ is the first positive zero of the Dini function $z \mapsto(2-\nu) J_{\nu}(z)+z J_{\nu}^{\prime}(z)$. Moreover, the function $\alpha \mapsto r_{\alpha, \beta}\left(h_{\nu}\right)$ is strictly decreasing on $[0, \infty)$ and consequently we have $r_{\beta}^{c}\left(h_{\nu}\right)<r_{\alpha, \beta}\left(h_{\nu}\right)<r_{\beta}^{*}\left(h_{\nu}\right)$ for all $\alpha \in(0,1), \beta \in[0,1)$ and $\nu>-1$.

Finally, we mention that the cases $\alpha=0$ and $\alpha=1$ of the above Theorem were also considered recently in [4, Theorem 1(c)] and [6, Theorem 1.3]. Our Theorem 3 is a common generalization of these results. Figure 3 illustrates the fact that for $\alpha \in[0,1]$ the radius of $\alpha$-convexity of the function $h_{\nu}$ is between its radii of convexity and starlikeness, that is, $r_{\beta}^{c}\left(h_{\nu}\right)<r_{\alpha, \beta}\left(h_{\nu}\right)<r_{\beta}^{*}\left(h_{\nu}\right)$ for all $\alpha \in(0,1), \beta \in[0,1)$ and $\nu>$ -1 . We considered the particular cases when $\beta=0.29, \nu=-0.5$ and $\alpha \in\{0,0.3,0.4,0.8,1\}$. We would like to take the opportunity to mention that [4, Theorem 1(c)] should be corrected as follows: if $\nu>-1$, then $r^{*}\left(h_{\nu}\right)=z_{\nu, \beta, 1}$, where $z_{\nu, \beta, 1}$ is the smallest positive root of the equation $z^{\frac{1}{2}} J_{\nu}^{\prime}\left(z^{\frac{1}{2}}\right)+(2-2 \beta-\nu) J_{\nu}\left(z^{\frac{1}{2}}\right)=0$. In [4, Theorem $1(\mathrm{c})$ ] the above result was stated wrongly with $z$ instead of $z^{\frac{1}{2}}$. Consequently, 44, Corollary $1(\mathrm{c})$ ] should be rewritten accordingly as follows: if $\nu>-1$, then the radius of starlikeness of the function $h_{\nu}$ is $z_{\nu, 0,1}$, which denotes the smallest positive root of the equation $z^{\frac{1}{2}} J_{\nu}^{\prime}\left(z^{\frac{1}{2}}\right)+(2-\nu) J_{\nu}\left(z^{\frac{1}{2}}\right)=0$. 


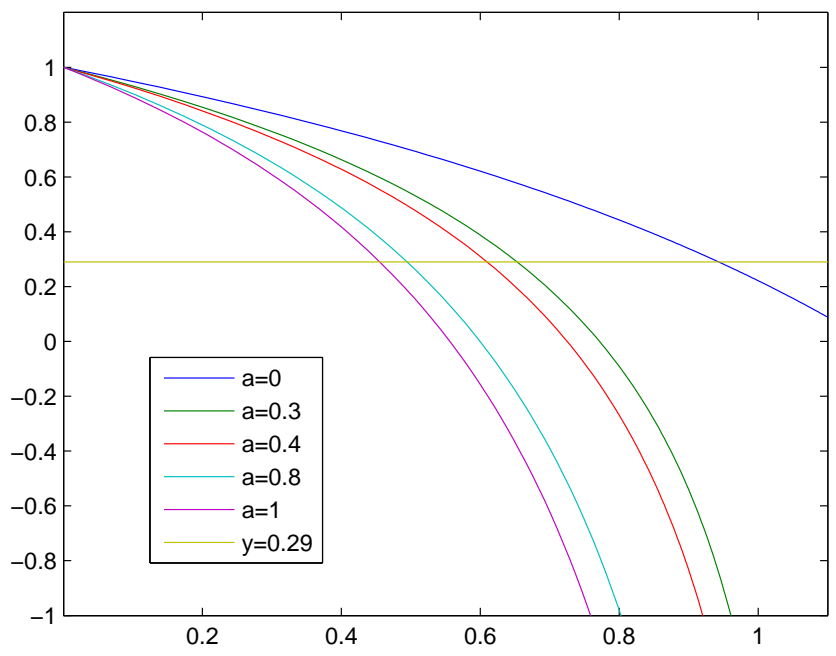

Figure 3. The graph of the function $\left.r \mapsto \mathbb{J}\left(a, h_{-0.5} r\right)\right)$ for $a \in\{0,0.3,0.4,0.8,1\}$ on $[0,1.1]$.

\section{Proof of the main results}

In this section our aim is to present the proofs of the main theorems. For convenience in the sequel we will use the following notation

$$
\mathbb{J}(\alpha, u(z))=(1-\alpha) \frac{z u^{\prime}(z)}{u(z)}+\alpha\left(1+\frac{z u^{\prime \prime}(z)}{u^{\prime}(z)}\right) .
$$

Proof of Theorem 1. Without loss of generality we assume that $\alpha>0$. The case $\alpha=0$ was proved already in [4]. By using the definition of the function $f_{\nu}$ we have

$$
\frac{z f_{\nu}^{\prime}(z)}{f_{\nu}(z)}=\frac{1}{\nu} \frac{z J_{\nu}^{\prime}(z)}{J_{\nu}(z)}, \quad 1+\frac{z f_{\nu}^{\prime \prime}(z)}{f_{\nu}^{\prime}(z)}=1+\frac{z J_{\nu}^{\prime \prime}(z)}{J_{\nu}^{\prime}(z)}+\left(\frac{1}{\nu}-1\right) \frac{z J_{\nu}^{\prime}(z)}{J_{\nu}(z)} .
$$

In view of the following infinite product representations [14, p. 235]

$$
J_{\nu}(z)=\frac{\left(\frac{1}{2} z\right)^{\nu}}{\Gamma(\nu+1)} \prod_{n \geq 1}\left(1-\frac{z^{2}}{j_{\nu, n}^{2}}\right), \quad J_{\nu}^{\prime}(z)=\frac{\left(\frac{1}{2} z\right)^{\nu-1}}{2 \Gamma(\nu)} \prod_{n \geq 1}\left(1-\frac{z^{2}}{j_{\nu, n}^{\prime 2}}\right),
$$

where $j_{\nu, n}$ and $j_{\nu, n}^{\prime}$ are the $n$th positive roots of $J_{\nu}$ and $J_{\nu}^{\prime}$, respectively, logarithmic differentiation yields

$$
\frac{z J_{\nu}^{\prime}(z)}{J_{\nu}(z)}=\nu-\sum_{n \geq 1} \frac{2 z^{2}}{j_{\nu, n}^{2}-z^{2}}, \quad 1+\frac{z J_{\nu}^{\prime \prime}(z)}{J_{\nu}^{\prime}(z)}=\nu-\sum_{n \geq 1} \frac{2 z^{2}}{j_{\nu, n}^{\prime 2}-z^{2}},
$$

which implies that

$$
\begin{aligned}
\mathbb{J}\left(\alpha, f_{\nu}(z)\right) & =(1-\alpha) \frac{z f_{\nu}^{\prime}(z)}{f_{\nu}(z)}+\alpha\left(1+\frac{z f_{\nu}^{\prime \prime}(z)}{f_{\nu}^{\prime}(z)}\right) \\
& =\alpha+\left(\frac{1}{\nu}-\alpha\right) \frac{z J_{\nu}^{\prime}(z)}{z J_{\nu}(z)}+\alpha \frac{z J_{\nu}^{\prime \prime}(z)}{J_{\nu}^{\prime}(z)} \\
& =1-\left(\frac{1}{\nu}-\alpha\right) \sum_{n \geq 1} \frac{2 z^{2}}{j_{\nu, n}^{2}-z^{2}}-\alpha \sum_{n \geq 1} \frac{2 z^{2}}{j_{\nu, n}^{\prime 2}-z^{2}} .
\end{aligned}
$$

On the other hand, we know (see [6, Lemma 2.1]) that if $a>b>0, z \in \mathbb{C}$ and $\lambda \leq 1$, then for all $|z|<b$ we have

$$
\lambda \operatorname{Re}\left(\frac{z}{a-z}\right)-\operatorname{Re}\left(\frac{z}{b-z}\right) \geq \lambda \frac{|z|}{a-|z|}-\frac{|z|}{b-|z|} .
$$

Note that in [6, Lemma 2.1] it was assumed that $\lambda \in[0,1]$, however, following the proof of [6. Lemma 2.1] it is clear that we do not need the assumption $\lambda \geq 0$. By using the inequality (2.1) for all $z \in \mathbb{D}\left(0, j_{\nu, 1}^{\prime}\right)$ 
we obtain the inequality

$$
\frac{1}{\alpha} \operatorname{Re} \mathbb{J}\left(\alpha, f_{\nu}(z)\right) \geq \frac{1}{\alpha}+\left(1-\frac{1}{\alpha \nu}\right) \sum_{n \geq 1} \frac{2 r^{2}}{j_{\nu, n}^{2}-r^{2}}-\sum_{n \geq 1} \frac{2 r^{2}}{j_{\nu, n}^{\prime 2}-r^{2}}=\frac{1}{\alpha} \mathbb{J}\left(\alpha, f_{\nu}(r)\right),
$$

where $|z|=r$. Here we used that the zeros $j_{\nu, n}$ and $j_{\nu, n}^{\prime}$ interlace according to the inequalities [14, p. 235]

$$
\nu \leq j_{\nu, 1}^{\prime}<j_{\nu, 1}<j_{\nu, 2}^{\prime}<j_{\nu, 2}<j_{\nu, 3}^{\prime}<\ldots
$$

Now, the above deduced inequality implies that for $r \in\left(0, j_{\nu, 1}^{\prime}\right)$ we have $\inf _{z \in \mathbb{D}(0, r)} \mathbb{J}\left(\alpha, f_{\nu}(z)\right)=\mathbb{J}\left(\alpha, f_{\nu}(r)\right)$. On the other hand, the function $r \mapsto \mathbb{J}\left(\alpha, f_{\nu}(r)\right)$ is strictly decreasing on $\left(0, j_{\nu, 1}^{\prime}\right)$ since

$$
\begin{aligned}
\frac{\partial}{\partial r} \mathbb{J}\left(\alpha, f_{\nu}(r)\right) & =-\left(\frac{1}{\nu}-\alpha\right) \sum_{n \geq 1} \frac{4 r j_{\nu, n}^{2}}{\left(j_{\nu, n}^{2}-r^{2}\right)^{2}}-\alpha \sum_{n \geq 1} \frac{4 r j_{\nu, n}^{\prime 2}}{\left(j_{\nu, n}^{\prime 2}-r^{2}\right)^{2}} \\
& <\alpha \sum_{n \geq 1} \frac{4 r j_{\nu, n}^{2}}{\left(j_{\nu, n}^{2}-r^{2}\right)^{2}}-\alpha \sum_{n \geq 1} \frac{4 r j_{\nu, n}^{\prime 2}}{\left(j_{\nu, n}^{\prime 2}-r^{2}\right)^{2}}<0
\end{aligned}
$$

for $\nu>0$ and $r \in\left(0, j_{\nu, 1}^{\prime}\right)$. Here we used again that the zeros $j_{\nu, n}$ and $j_{\nu, n}^{\prime}$ interlace and for all $n \in \mathbb{N}$, $\nu>0$ and $r<\sqrt{j_{\nu, 1} j_{\nu, 1}^{\prime}}$ we have that

$$
j_{\nu, n}^{2}\left(j_{\nu, n}^{\prime 2}-r^{2}\right)^{2}<j_{\nu, n}^{\prime 2}\left(j_{\nu, n}^{2}-r^{2}\right)^{2}
$$

We also have that $\lim _{r \searrow 0} \mathbb{J}\left(\alpha, f_{\nu}(r)\right)=1>\beta$ and $\lim _{r} \nearrow_{\nu, 1}^{\prime} \mathbb{J}\left(\alpha, f_{\nu}(r)\right)=-\infty$, which means that for $z \in \mathbb{D}\left(0, r_{1}\right)$ we have $\operatorname{Re} \mathbb{J}\left(\alpha, f_{\nu}(z)\right)>\beta$ if and only if $r_{1}$ is the unique root of $\mathbb{J}\left(\alpha, f_{\nu}(r)\right)=\beta$, situated in $\left(0, j_{\nu, 1}^{\prime}\right)$. Finally, by using again the interlacing inequalities (2.2) we obtain the inequality

$$
\frac{\partial}{\partial \alpha} \mathbb{J}\left(\alpha, f_{\nu}(r)\right)=\sum_{n \geq 1} \frac{2 r^{2}}{j_{\nu, n}^{2}-r^{2}}-\sum_{n \geq 1} \frac{2 r^{2}}{j_{\nu, n}^{\prime 2}-r^{2}}<0
$$

where $\nu>0, \alpha \geq 0$ and $r \in\left(0, j_{\nu, 1}^{\prime}\right)$. This implies that the function $\alpha \mapsto \mathbb{J}\left(\alpha, f_{\nu}(r)\right)$ is strictly decreasing on $[0, \infty)$ for all $\nu>0$ and $r \in\left(0, j_{\nu, 1}^{\prime}\right)$ fixed. Consequently, as a function of $\alpha$ the unique root of the equation $\mathbb{J}\left(\alpha, f_{\nu}(r)\right)=\beta$ is strictly decreasing, where $\beta \in[0,1), \nu>0$ and $r \in\left(0, j_{\nu, 1}^{\prime}\right)$ are fixed. Thus, in the case when $\alpha \in(0,1)$ the radius of $\alpha$-convexity of the function $f_{\nu}$ will be between the radius of convexity and the radius of starlikeness of the function $f_{\nu}$. This completes the proof.

Proof of Theorem 2. Similarly, as in the proof of Theorem 1 we assume that $\alpha>0$. The case $\alpha=0$ was proved already in [4. We start with the following relations

$$
\frac{z g_{\nu}^{\prime}(z)}{g_{\nu}(z)}=1-\nu+\frac{z J_{\nu}^{\prime}(z)}{J_{\nu}(z)}, \quad z \frac{g_{\nu}^{\prime \prime}(z)}{g_{\nu}^{\prime}(z)}=\frac{\nu(\nu-1) J_{\nu}(z)+2(1-\nu) z J_{\nu}^{\prime}(z)+z^{2} J_{\nu}^{\prime \prime}(z)}{(1-\nu) J_{\nu}(z)+z J_{\nu}^{\prime}(z)} .
$$

The recurrence formula [14, p. 222] $z J_{\nu}^{\prime}(z)=\nu J_{\nu}(z)-z J_{\nu+1}(z)$ and the fact that $J_{\nu}$ is a particular solution of the Bessel differential equation imply that

$$
z \frac{g_{\nu}^{\prime \prime}(z)}{g_{\nu}^{\prime}(z)}=z \frac{z J_{\nu+2}(z)-3 J_{\nu+1}(z)}{J_{\nu}(z)-z J_{\nu+1}(z)}
$$

and using [6. Lemma 2.4] it follows that

$$
1+z \frac{g_{\nu}^{\prime \prime}(z)}{g_{\nu}^{\prime}(z)}=1-\sum_{n \geq 1} \frac{2 z^{2}}{\alpha_{\nu, n}^{2}-z^{2}}
$$

where $\alpha_{\nu, n}$ is the $n$th positive zero of the Dini function $z \mapsto(1-\nu) J_{\nu}(z)+z J_{\nu}^{\prime}(z)$. Thus, we have that

$$
\begin{aligned}
\mathbb{J}\left(\alpha, g_{\nu}(z)\right) & =(1-\alpha) \frac{z g_{\nu}^{\prime}(z)}{g_{\nu}(z)}+\alpha\left(1+\frac{z g_{\nu}^{\prime \prime}(z)}{g_{\nu}^{\prime}(z)}\right) \\
& =(1-\alpha)\left(1-\nu+\frac{z J_{\nu}^{\prime}(z)}{J_{\nu}(z)}\right)+\alpha\left(1+z \frac{z J_{\nu+2}(z)-3 J_{\nu+1}(z)}{J_{\nu}(z)-z J_{\nu+1}(z)}\right) \\
& =1+(\alpha-1) \sum_{n \geq 1} \frac{2 z^{2}}{j_{\nu, n}^{2}-z^{2}}-\alpha \sum_{n \geq 1} \frac{2 z^{2}}{\alpha_{\nu, n}^{2}-z^{2}} .
\end{aligned}
$$


Applying the inequality (2.1) we have that

$$
\frac{1}{\alpha} \operatorname{Re} \mathbb{J}\left(\alpha, g_{\nu}(z)\right) \geq \frac{1}{\alpha}+\left(1-\frac{1}{\alpha}\right) \sum_{n \geq 1} \frac{2 r^{2}}{j_{\nu, n}^{2}-r^{2}}-\sum_{n \geq 1} \frac{2 r^{2}}{\alpha_{\nu, n}^{2}-r^{2}}=\frac{1}{\alpha} \mathbb{J}\left(\alpha, g_{\nu}(r)\right),
$$

where $|z|=r$. Here we used tacitly that for all $n \in\{1,2, \ldots\}$ we have $\alpha_{\nu, n} \in\left(j_{\nu, n-1}, j_{\nu, n}\right)$, where $j_{\nu, n}$ is the $n$th positive zero of $J_{\nu}$. This follows immediately from Dixon's theorem [17, p. 480], which says that when $\nu>-1$ and $a, b, c, d$ are constants such that $a d \neq b c$, then the positive zeros of $z \mapsto a J_{\nu}(z)+b z J_{\nu}^{\prime}(z)$ are interlaced with those of $z \mapsto c J_{\nu}(z)+d z J_{\nu}^{\prime}(z)$. Thus, if we choose $a=1-\nu, b=1, c=1$ and $d=0$, then the required assertion follows. Note also that the zeros $\alpha_{\nu, n}$ are all real when $\nu>-1$, see [17, p. 482], and thus the application of the inequality (2.1) is allowed. Thus, for $r \in\left(0, \alpha_{\nu, 1}\right)$ we get $\inf _{z \in \mathbb{D}(0, r)} \operatorname{Re} \mathbb{J}\left(\alpha, g_{\nu}(z)\right)=\mathbb{J}\left(\alpha, g_{\nu}(r)\right)$, since according to the minimum principle of harmonic functions the infimum is taken on the boundary. On the other hand, the function $r \mapsto \mathbb{J}\left(\alpha, g_{\nu}(r)\right)$ is strictly decreasing on $\left(0, \alpha_{\nu, 1}\right)$ since

$$
\begin{aligned}
\frac{\partial}{\partial r} \mathbb{J}\left(\alpha, g_{\nu}(r)\right) & =(\alpha-1) \sum_{n \geq 1} \frac{4 r j_{\nu, n}^{2}}{\left(j_{\nu, n}^{2}-r^{2}\right)^{2}}-\alpha \sum_{n \geq 1} \frac{4 r \alpha_{\nu, n}^{2}}{\left(\alpha_{\nu, n}^{2}-r^{2}\right)^{2}} \\
& <\alpha \sum_{n \geq 1} \frac{4 r j_{\nu, n}^{2}}{\left(j_{\nu, n}^{2}-r^{2}\right)^{2}}-\alpha \sum_{n \geq 1} \frac{4 r \alpha_{\nu, n}^{2}}{\left(\alpha_{\nu, n}^{2}-r^{2}\right)^{2}}<0
\end{aligned}
$$

for $\nu>-1$ and $r \in\left(0, \alpha_{\nu, 1}\right)$. Here we used again that the zeros $j_{\nu, n}$ and $\alpha_{\nu, n}$ interlace and for all $n \in \mathbb{N}$, $\nu>-1$ and $r<\sqrt{j_{\nu, 1} \alpha_{\nu, 1}}$ we have that

$$
j_{\nu, n}^{2}\left(\alpha_{\nu, n}^{2}-r^{2}\right)^{2}<\alpha_{\nu, n}^{2}\left(j_{\nu, n}^{2}-r^{2}\right)^{2} .
$$

We also have that $\lim _{r \searrow 0} \mathbb{J}\left(\alpha, g_{\nu}(r)\right)=1>\beta$ and $\lim _{r} \gamma_{\nu, 1} \mathbb{J}\left(\alpha, g_{\nu}(r)\right)=-\infty$, which means that for $z \in \mathbb{D}\left(0, r_{2}\right)$ we have $\operatorname{Re} \mathbb{J}\left(\alpha, g_{\nu}(z)\right)>\beta$ if and only if $r_{2}$ is the unique root of $\mathbb{J}\left(\alpha, g_{\nu}(r)\right)=\beta$, situated in $\left(0, \alpha_{\nu, 1}\right)$. Finally, by using again the interlacing inequalities $j_{\nu, n-1}<\alpha_{\nu, n}<j_{\nu, n}$ we obtain the inequality

$$
\frac{\partial}{\partial \alpha} \mathbb{J}\left(\alpha, g_{\nu}(r)\right)=\sum_{n \geq 1} \frac{2 r^{2}}{j_{\nu, n}^{2}-r^{2}}-\sum_{n \geq 1} \frac{2 r^{2}}{\alpha_{\nu, n}^{2}-r^{2}}<0
$$

where $\nu>-1, \alpha \geq 0$ and $r \in\left(0, \alpha_{\nu, 1}\right)$. This implies that the function $\alpha \mapsto \mathbb{J}\left(\alpha, g_{\nu}(r)\right)$ is strictly decreasing on $[0, \infty)$ for all $\nu>-1$ and $r \in\left(0, \alpha_{\nu, 1}\right)$ fixed. Consequently, as a function of $\alpha$ the unique root of the equation $\mathbb{J}\left(\alpha, g_{\nu}(r)\right)=\beta$ is strictly decreasing, where $\beta \in[0,1), \nu>-1$ and $r \in\left(0, \alpha_{\nu, 1}\right)$ are fixed. Thus, for $\alpha \in(0,1)$ the radius of $\alpha$-convexity of the function $g_{\nu}$ is between the radius of convexity and the radius of starlikeness of the function $g_{\nu}$.

Proof of Theorem 3. Similarly, as in the proof of Theorems 1 and 2 we assume that $\alpha>0$. The case $\alpha=0$ was proved already in [4]. Combining

$$
\frac{z h_{\nu}^{\prime}(z)}{h_{\nu}(z)}=1-\frac{\nu}{2}+\frac{1}{2} \frac{z^{\frac{1}{2}} J_{\nu}^{\prime}\left(z^{\frac{1}{2}}\right)}{J_{\nu}\left(z^{\frac{1}{2}}\right)}=1-\sum_{n \geq 1} \frac{z}{j_{\nu, n}^{2}-z}
$$

with [6, Lemma 2.5]

$$
z \frac{h_{\nu}^{\prime \prime}(z)}{h_{\nu}^{\prime}(z)}=\frac{\nu(\nu-2) J_{\nu}\left(z^{\frac{1}{2}}\right)+(3-2 \nu) z^{\frac{1}{2}} J_{\nu}^{\prime}\left(z^{\frac{1}{2}}\right)+z J_{\nu}^{\prime \prime}\left(z^{\frac{1}{2}}\right)}{2(2-\nu) J_{\nu}\left(z^{\frac{1}{2}}\right)+2 z^{\frac{1}{2}} J_{\nu}^{\prime}\left(z^{\frac{1}{2}}\right)}=-\sum_{n \geq 1} \frac{z}{\beta_{\nu, n}^{2}-z}
$$

where $\beta_{\nu, n}$ stands for the $n$th positive zero of the Dini function $z \mapsto(2-\nu) J_{\nu}(z)+z J_{\nu}^{\prime}(z)$, it follows that

$$
\mathbb{J}\left(\alpha, h_{\nu}(z)\right)=(1-\alpha) \frac{z h_{\nu}^{\prime}(z)}{h_{\nu}(z)}+\alpha\left(1+\frac{z h_{\nu}^{\prime \prime}(z)}{h_{\nu}^{\prime}(z)}\right)=1+(\alpha-1) \sum_{n \geq 1} \frac{z}{j_{\nu, n}^{2}-z}-\alpha \sum_{n \geq 1} \frac{z}{\beta_{\nu, n}^{2}-z} .
$$

Applying again the inequality (2.1) we have that

$$
\frac{1}{\alpha} \operatorname{Re} \mathbb{J}\left(\alpha, h_{\nu}(z)\right) \geq \frac{1}{\alpha}+\left(1-\frac{1}{\alpha}\right) \sum_{n \geq 1} \frac{r}{j_{\nu, n}^{2}-r}-\sum_{n \geq 1} \frac{r}{\beta_{\nu, n}^{2}-r}=\frac{1}{\alpha} \mathbb{J}\left(\alpha, h_{\nu}(r)\right),
$$

where $|z|=r$. Here we used tacitly that for all $n \in\{1,2, \ldots\}$ we have $\beta_{\nu, n} \in\left(j_{\nu, n-1}, j_{\nu, n}\right)$, which follows immediately from Dixon's theorem [17, p. 480], similarly as in the case the roots $\alpha_{\nu, n}$ in the proof of Theorem 2. Note also that the zeros $\beta_{\nu, n}$ are all real when $\nu>-1$, see [17, p. 482], and thus the 
application of the inequality (2.1) is allowed. Thus, for $r \in\left(0, \beta_{\nu, 1}^{2}\right)$ we get $\inf _{z \in \mathbb{D}(0, r)} \operatorname{Re} \mathbb{J}\left(\alpha, h_{\nu}(z)\right)=$ $\mathbb{J}\left(\alpha, h_{\nu}(r)\right)$. On the other hand, the function $r \mapsto \mathbb{J}\left(\alpha, h_{\nu}(r)\right)$ is strictly decreasing on $\left(0, \beta_{\nu, 1}^{2}\right)$ since

$$
\begin{aligned}
\frac{\partial}{\partial r} \mathbb{J}\left(\alpha, h_{\nu}(r)\right) & =(\alpha-1) \sum_{n \geq 1} \frac{r j_{\nu, n}^{2}}{\left(j_{\nu, n}^{2}-r\right)^{2}}-\alpha \sum_{n \geq 1} \frac{r \beta_{\nu, n}^{2}}{\left(\beta_{\nu, n}^{2}-r\right)^{2}} \\
& <\alpha \sum_{n \geq 1} \frac{r j_{\nu, n}^{2}}{\left(j_{\nu, n}^{2}-r\right)^{2}}-\alpha \sum_{n \geq 1} \frac{r \beta_{\nu, n}^{2}}{\left(\beta_{\nu, n}^{2}-r\right)^{2}}<0
\end{aligned}
$$

for $\nu>-1$ and $r \in\left(0, \beta_{\nu, 1}^{2}\right)$. Here we used again that the zeros $j_{\nu, n}$ and $\beta_{\nu, n}$ interlace and for all $n \in \mathbb{N}$, $\nu>-1$ and $r<j_{\nu, 1} \beta_{\nu, 1}$ we have that

$$
j_{\nu, n}^{2}\left(\beta_{\nu, n}^{2}-r\right)^{2}<\beta_{\nu, n}^{2}\left(j_{\nu, n}^{2}-r\right)^{2} .
$$

We also have that $\lim _{r \succeq 0} \mathbb{J}\left(\alpha, h_{\nu}(r)\right)=1>\beta$ and $\lim _{r} \gamma_{\nu, 1} \mathbb{J}\left(\alpha, g_{\nu}(r)\right)=-\infty$, which means that for $z \in \mathbb{D}\left(0, r_{3}\right)$ we have $\operatorname{Re} \mathbb{J}\left(\alpha, h_{\nu}(z)\right)>\beta$ if and only if $r_{3}$ is the unique root of $\mathbb{J}\left(\alpha, h_{\nu}(r)\right)=\beta$, situated in $\left(0, \beta_{\nu, 1}^{2}\right)$. Finally, by using again the interlacing inequalities $j_{\nu, n-1}<\beta_{\nu, n}<j_{\nu, n}$ we obtain the inequality

$$
\frac{\partial}{\partial \alpha} \mathbb{J}\left(\alpha, h_{\nu}(r)\right)=\sum_{n \geq 1} \frac{r}{j_{\nu, n}^{2}-r}-\sum_{n \geq 1} \frac{r}{\beta_{\nu, n}^{2}-r}<0,
$$

where $\nu>-1, \alpha \geq 0$ and $r \in\left(0, \beta_{\nu, 1}^{2}\right)$. This implies that the function $\alpha \mapsto \mathbb{J}\left(\alpha, h_{\nu}(r)\right)$ is strictly decreasing on $[0, \infty)$ for all $\nu>-1$ and $r \in\left(0, \beta_{\nu, 1}^{2}\right)$ fixed. Consequently, as a function of $\alpha$ the unique root of the equation $\mathbb{J}\left(\alpha, h_{\nu}(r)\right)=\beta$ is strictly decreasing, where $\beta \in[0,1), \nu>-1$ and $r \in\left(0, \beta_{\nu, 1}^{2}\right)$ are fixed. Thus, when $\alpha \in(0,1)$ the radius of $\alpha$-convexity of the function $h_{\nu}$ is between the radius of convexity and the radius of starlikeness of the function $h_{\nu}$.

\section{REFERENCES}

[1] Á. BARICZ, Geometric properties of generalized Bessel functions of complex order, Mathematica 48(71) (2006) 13-18.

[2] Á. BARICZ, Geometric properties of generalized Bessel functions, Publ. Math. Debrecen 73 (2008) 155-178.

[3] Á. BARICZ, Generalized Bessel Functions of the First Kind, Lecture Notes in Mathematics, vol. 1994, Springer-Verlag, Berlin, 2010

[4] Á. Baricz, P.A. Kupán, R. SzÁsz, The radius of starlikeness of normalized Bessel functions of the first kind, Proc. Amer. Math. Soc. 142(6) (2014) 2019-2025.

[5] Á. Baricz, S. Ponnusamy, Starlikeness and convexity of generalized Bessel functions, Integr. Transforms Spec. Funct. 21 (2010) 641-653.

[6] Á. BARICZ, R. SzÁsz, The radius of convexity of normalized Bessel functions of the first kind, Anal. Appl. 12(5) (2014) 485-509.

[7] R.K. Brown, Univalence of Bessel functions, Proc. Amer. Math. Soc. 11(2) (1960) 278-283.

[8] R.K. Brown, Univalent solutions of $W^{\prime \prime}+p W=0$, Canad. J. Math. 14 (1962) 69-78.

[9] R.K. Brown, Univalence of normalized solutions of $W^{\prime \prime}(z)+p(z) W(z)=0$, Int. J. Math. Math. Sci. 5 (1982) $459-483$.

[10] P.L. Duren, Univalent Functions, Grundlehren Math.Wiss. 259, Springer, New York, 1983.

[11] E. Kreyszig, J. Todd, The radius of univalence of Bessel functions, Illinois J. Math. 4 (1960) 143-149.

[12] P. Mocanu, Une propriété de convexité généralisée dans la théorie de la représentation conforme, Mathematica 11(34) (1969) 127-133.

[13] P.T. Mocanu, M.O. Reade, The radius of $\alpha$-convexity for the class of starlike univalent functions, $\alpha$ real, Proc. Amer. Math. Soc. 51(2) (1975) 395-400.

[14] F.W.J. Olver, D.W. Lozier, R.F. Boisvert, C.W. Clark (Eds.), NIST Handbook of Mathematical Functions, Cambridge Univ. Press, Cambridge, 2010.

[15] R. SzÁsz, On starlikeness of Bessel functions of the first kind, In: Proceedings of the 8th Joint Conference on Mathematics and Computer Science, Komárno, Slovakia, 2010, 9pp.

[16] R. SzÁsz, P.A. Kupán, About the univalence of the Bessel functions, Stud. Univ. Babeş-Bolyai Math. 54(1) (2009) $127-132$.

[17] G.N. Watson, A Treatise of the Theory of Bessel Functions, Cambridge University Press, Cambridge, 1995.

Department of Economics, Babeş-Bolyai University, 400591 Cluj-Napoca, Romania

Institute of Applied Mathematics, Óbuda University, 1034 Budapest, Hungary

E-mail address: bariczocsi@yahoo.com

Department of Mathematics, Ataturk University, 25240 Erzurum, Turkey

E-mail address: horhan@atauni.edu.tr

Department of Mathematics and Informatics, Sapientia Hungarian University of Transylvania, 540485 TÂrgu-

Mureş, Romania

E-mail address: rszasz@ms.sapientia.ro 